\title{
SUPPRESSION AND TREATMENT OF HALOPERIDOL INDUCED EXTRA- PYRAMIDAL SIDE EFFECTS AND ANXIETY SYNDROME BY THE COADMINISTERATION OF RED RICE BRAN OIL IN RATS.
}

\author{
Farah Naz \& Erum Shireen. \\ Department of Biochemistry, University of Karachi \\ Corresponding Author Email: gummygurl_24@hotmail.com
}

ABSTRACT

Antipsychotic medications may reduce or eliminate symptoms of psychosis. They are not a 'cure' for mental illnesses, but can be an effective part of treating mental disorders. Even though many of the antipsychotics such as haloperidol cannot cure mental illnesses, they are effective in eliminating or reducing psychotic symptoms. However, prolong administration of haloperidol may result in parkinsonian like effects and tardive dyskinesia. The objective of the present research was to determine the effects of long term co-administration of RRBO in diet on haloperidol induced anxiety and EPS. It was hypothesized that co-administration of RRBO by the free radicals scavenging property could alleviate parkinsonian like effects and tardive dyskinesia. The present results showed a reversal of haloperidol induced parkinsonian like effects and tardive dyskinesia in rats cotreated with RRBO.

\section{KEYWORDS}

Antidepressants, Antipsychotics, Haloperidol, Tardive dyskinesia, Schizophrenia, Parkinson's disease, Red rice bran oil, Vacuous chewing movements.

\section{INTRODUCTION}

The antipsychotic drugs, which are essentially dopamine receptor blockers, are used to treat disorders that include thought processes. Such kinds of medications are referred to as neuroleptic agents because of their inter-related neurological undesirable effects (Dilsaver, 1988).

Haloperidol belongs to the typical antipsychotic class; containing butyrophenone and moreover it has also similar effects as that of phenothiazine i.e. especially from the pharmacological aspect. It belongs to the second generation antipsychotics. Second generation antipsychotic agents (SGAs), more commonly known as atypical APs (AAPs), which do a lot more than FGAs i.e. they are broad-spectrum antagonists of alpha-noradrenergic, dopamine, and serotonin receptors (Poore, 2010). Haloperidol is used in the treatment of schizophrenia and, more acutely, in the treatment of acute psychotic states. Haloperidol is occasionally called vitamin H.A comprehensive review of haloperidol has found it to be an effective agent in treatment of symptoms associated with schizophrenia (David, 1998).

\section{What is schizophrenia?}

Schizophrenia is a chronic, severe, and disabling mental illness. It affects men and women with equal frequency. People suffering from schizophrenia may have the following symptoms: Delusions, Hallucinations, Disorganized thoughts and behavior, disorganized speech and catatonic behavior. In medical terms, schizophrenia refers to a split-personality disorder (Tuthill, 2006). Since the introduction of the second generation or atypical antipsychotics (AAP), these agents have been widely prescribed for the management of patients with schizophrenia, bipolar disorders, other psychotic disorders or conditions with severe behavioral disturbance. The increasing use of AAP is in part due to their lower propensity to induce extrapyramidal symptoms and tardive dyskinesia compared to typical antipsychotics (Sartorius, 2007).

What is tardive dyskinesia?

Tardive dyskinesia is a difficult-to-treat form of dyskinesia, a disorder resulting in involuntary, repetitive body movements. In this form of dyskinesia, the involuntary movements are tardive, meaning they have a slow or belated onset. This neurological disorder frequently appears after long-term or high-dose use of antipsychotic drugs, or in children and infants as a side effect from usage of drugs for gastrointestinal disorders. It is a serious side effect that occurs when you take medications called neuroleptics (Peter, 1999). The symptoms may include: facial grimacing, finger movements, jaw swinging, repetitive chewing and tongue thrusting.

\section{Parkinsonian like symptoms}

Parkinsonism (also known as Parkinsonian syndrome, atypical Parkinson's or secondary Parkinson's) is a neurological syndrome characterized by tremor, hypokinesia, rigidity and postural instability. The underlying causes of parkinsonian like symptoms are numerous and diagnosis can be complex (Addington, 2007).

\section{What is red rice bran oil?}

Rice bran oil (also known as rice bran extract) is the oil extracted from the germ and inner husk of red rice. Supplies of red rice bran oil are obtained mostly from Bhutan, Himalayan red rice and North America (Raisu presentation). Health benefits of red rice bran oil includes: enhancing natural beauty, increases energy, helps in losing weight, boosts immunity, lowers cancer risk, lowers cholesterol and heart diseases, improves eyesight, regulates blood sugar levels, reduces headache, improves mental function and decreases kidney stones. Scientists in Japan discovered that Red rice bran contains over 2000+ antioxidants including 120 known 
super antioxidants, cofactors and catalysts-including: tocotrienols, tocopherols, phytosterols, CoQ10, alpha lipoic acid, IP-6, lutein, glutathione, carotenoids, selenium, lycopenes, gamma oryzanol and omega 3,6 and 9. Red rice bran extract has a myriad of nutrients that offers a perfect combination of detoxifying agents and brain boosters.

\section{MATERIALS AND METHODS}

Following material and methods were used in this study.

\section{Animals}

Locally bred male Albino-Wistar rats (Purchase from Dow University of Health Sciences) of weight 180-230 g were housed individually under 12-h light and dark cycle and controlled room temperature $\left(22 \pm 2.0^{\circ} \mathrm{C}\right)$ with free access to cubes of standard rodent diet and water for at least 3 days before experimentation.

\section{Drugs}

The drugs and oil used during the study were Haloperidol and Red rice bran oil. Red rice bran sample was provided from H.E.J Research Institute, University of Karachi. Haloperidol drops were given in drinking water for 5 weeks at clinically recommended dose. Haloperidol (Dosik, Adamjee Pharmaceutical Pvt. Ltd) purchase as oral drops of $2 \mathrm{mg} / \mathrm{ml}$ was given orally in drinking water in clinically recommended $(10 \mathrm{mg} / 70 \mathrm{~kg} /$ day $)$ dose. The drug was added in the drinking water at a concentration of $0.3 \mathrm{mg} / 100 \mathrm{ml}$. Average intake of water was $23 \pm 0.2 \mathrm{ml} / \mathrm{rat} /$ day and drug was $0.069 \mathrm{mg} \pm 0.02 / \mathrm{rat} / \mathrm{day}$. The animals were orally administered with saline or oil/day at 10 to 11 am for 5 weeks as described by (Marchese, 2002)

\section{BEHAVIORAL METHODS}

Behavioral methods used were;

\section{Open field activity test}

The assessment of locomotor activity and exploration in a novel environment as it may be altered by drug administration was done by open field test. The test consist of measuring the activity of rats in an open novel space, from which escape, is prevented by a surrounding wall (Haleem, 1996; Haleem, 2007). The open field apparatus used in the present investigation consisted of a square area $76 \times 76 \mathrm{~cm}$ with walls $42 \mathrm{~cm}$ high. The floor was divided by lines into 25 equal squares. To determine the activity, a rat was placed in the center square of the open field. The numbers of squares crossed with all four paws were scored for $5 \mathrm{~min}$.

\section{Elevated plus maze test}

The assessment of locomotor activity and exploration in a novel environment as it may be altered by drug administration was done by elevated plus maze test. The test setting consist of a plus-shaped apparatus with two open and two closed arms, each with an open roof, elevated $40-70 \mathrm{~cm}$ from the floor. To perform the test, an animal is exposed to the center of the plus on the elevated maze and number of entries in the open arm and time spent in the open arm is monitored for a fixed interval of time, preferably $5 \mathrm{~min}$ (Haleem, 2010)

\section{Light dark transition test}

The assessment of locomotor activity and exploration in a novel environment as it may be altered by drug administration was done by light dark transition test. The test is conducted in a two compartment light-dark box. Typical dimension of the two compartments are $26 \times 26 \times 26 \mathrm{~cm}$ for rats. The two compartments of equal size differ only in the sensory properties. One compartment is light and the other dark. The opening between the two compartments is typically not more than $7 \times 7 \mathrm{~cm}$. to determine the activity, a rat was placed in the light compartment of the light-dark box and the number of entries in to the light compartment and the time passed in to the light compartment is monitored for a fixed interval of time, preferably 5 min (Haleem, 2010).

\section{Home cage activity test}

The assessment of locomotor activity and exploration in a familiar environment as it may be altered by drug administration was done by home cage activity test.

The apparatus used in this study is a rectangular Perspex activity cage consisted of small square are $(26 \times 26 \times 26 \mathrm{~cm})$ with sawdustcovered floor. Testing was performed in a quiet room under white light as described by (Haleem, 2007). Before monitoring the activity, an animal was placed in it for 15 minute for habituation. Numbers of crossings across the box were monitored for $10 \mathrm{~min}$.

\section{Quantification of orofacial dyskinesia}

Animals placed individually in a rectangular Perspex activity cage $(26 \times 26 \times 26 \mathrm{~cm})$ with saw-dust floor were allowed to adapt to the observation cage for a period of 15 minutes. Orofacial dyskinesia was quantified as tardive VCMs during a 10 min observation period. A tardive VCM referred to the opening of mouth in the vertical plane with twitching of facial musculature and tongue protrusions, not directed towards physical materials.

\section{Monitoring catalepsy}

The cataleptic and anticataleptic effects of drugs were monitored on an inclined surface as describe before (Haleem, 2002). An animal to be tested was placed on the surface $\left(26^{*} 40 \mathrm{~cm}, 60^{\circ}\right.$ inclination) for a cut-off time of 3 minutes control rats changed their position immediately on the inclination. The latency to movement on the inclination was taken as a measure of catalepsy. Animals not moving on the inclination for a cut-off time $3 \mathrm{~min}$ were returned to their home cages. The catalepsy score was calculated as \{latency to movement (sec)/ cut off time (180sec) \} 100 .

\section{Statistical analysis}

SPSS 13 software was used for statistical analysis. Effects on behavior were analyzed by three-way ANOVA. Post hoc comparisons were carried out by Newman keuls test. $\mathrm{P}<0.05$ was taken as significant. Data is presented by means \pm S.D. 


\section{RESULTS}

Post hoc analysis by Newman Kuels test showed that when rats were exposed to novel environment in an open field box RRBO alone showed no change in latency to move (LTM) over control values during 5 weeks of administration. Haloperidol significantly decreases the LTM after $2^{\text {nd }}$ week and normalizes it after $5^{\text {th }}$ week of treatment. The co-administration of haloperidol and RRBO decreased LTM significantly after $1^{\text {st }}$ week but remain unaltered after $2^{\text {nd }}$ week.
Figure 4.1 shows the time course effect of red rice bran oil on haloperidol induced deficits in an open field. Three way ANOVA showed that significant effect of weeks $(\mathrm{F}=25.348, \mathrm{p}<0.01$, $\mathrm{df}=4$, 12) and Haloperidol $(\mathrm{F}=3.261$, $\mathrm{p}<0.05$, df 8,12$)$. The effects of RRBO (F=1.174, df 8,12, NS), interaction between weeks*Haloperidol ( $\mathrm{F}=2.391$, df 4,12, NS), weeks*RRBO $(\mathrm{F}=0.304$, df 4,12, NS), Halo*RRBO ( $\mathrm{F}=3.261$, df 4,12, NS) and the interaction between weeks*Halo*RRBO $(\mathrm{F}=2.391$, df 4,12 , NS) were not significant.
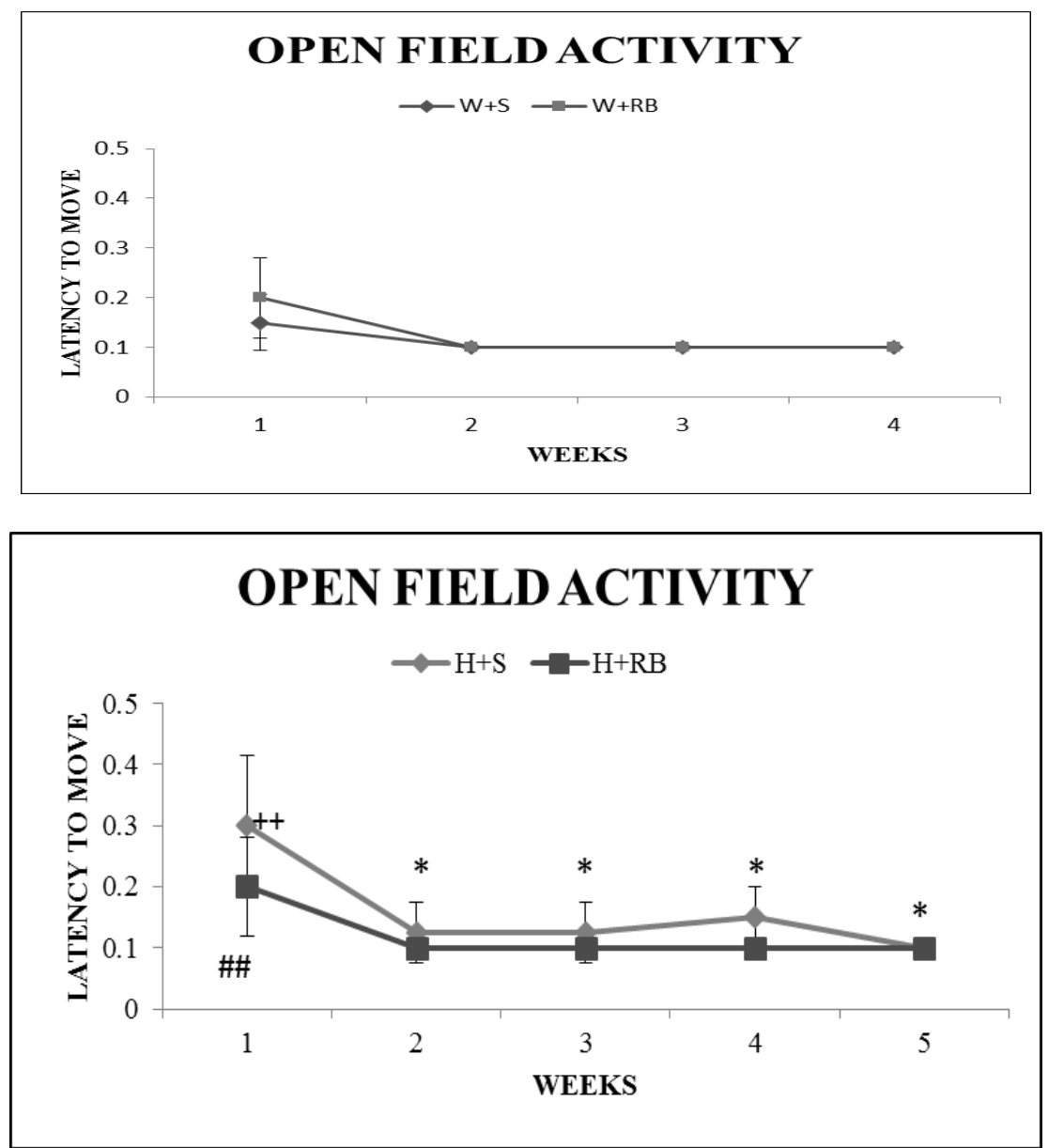

Fig 4.1(a\&b) time course effect of red rice bran oil on haloperidol induced deficits of exploratory activity in an open field

Values are means \pm S.D. $(\mathrm{n}=12)$. Significant differences by Newman-kuels test. $* p<0.05$ and $* * p<0.01$ from values of similarly treated animals, $+p<0.05$ and $++p<0.01$ from respective week values of water treated animals and \#p<0.05 and \#\#p<0.01 from respective week values of water+saline and haloperidol+saline treated animals.

Fig 4.2 (a\&b) shows the time-course effects of RRBO on haloperidol-induced deficits of exploratory activity in an open field. Three way ANOVA revealed the significant effects of weeks $(\mathrm{F}=7.847, \mathrm{p}<0.01$, df 4, 12), Halo $(\mathrm{F}=3.669, \mathrm{p}<0.05$, df 8,12) and
RRBO ( $\mathrm{F}=8.244, \mathrm{p}<0.01$, df 8,12) whereas non-significant effects were observed in weeks*Halo ( $\mathrm{F}=2.096$, df 4,12, NS), weeks*RRBO ( $\mathrm{F}=1.472$, df 4,12, NS) and Halo*RRBO $(\mathrm{F}=2.456$, df $4,12, \mathrm{NS}$ )

Post hoc analysis by Newman Kuels test showed that oral administration of RRBO significantly increased the exploratory activity during the $2^{\text {nd }}$ week but was not altered after that. Haloperidol and the co-administration of RRBO and Haloperidol did not alter the no. of squares crossed during the 5 weeks' treatment 

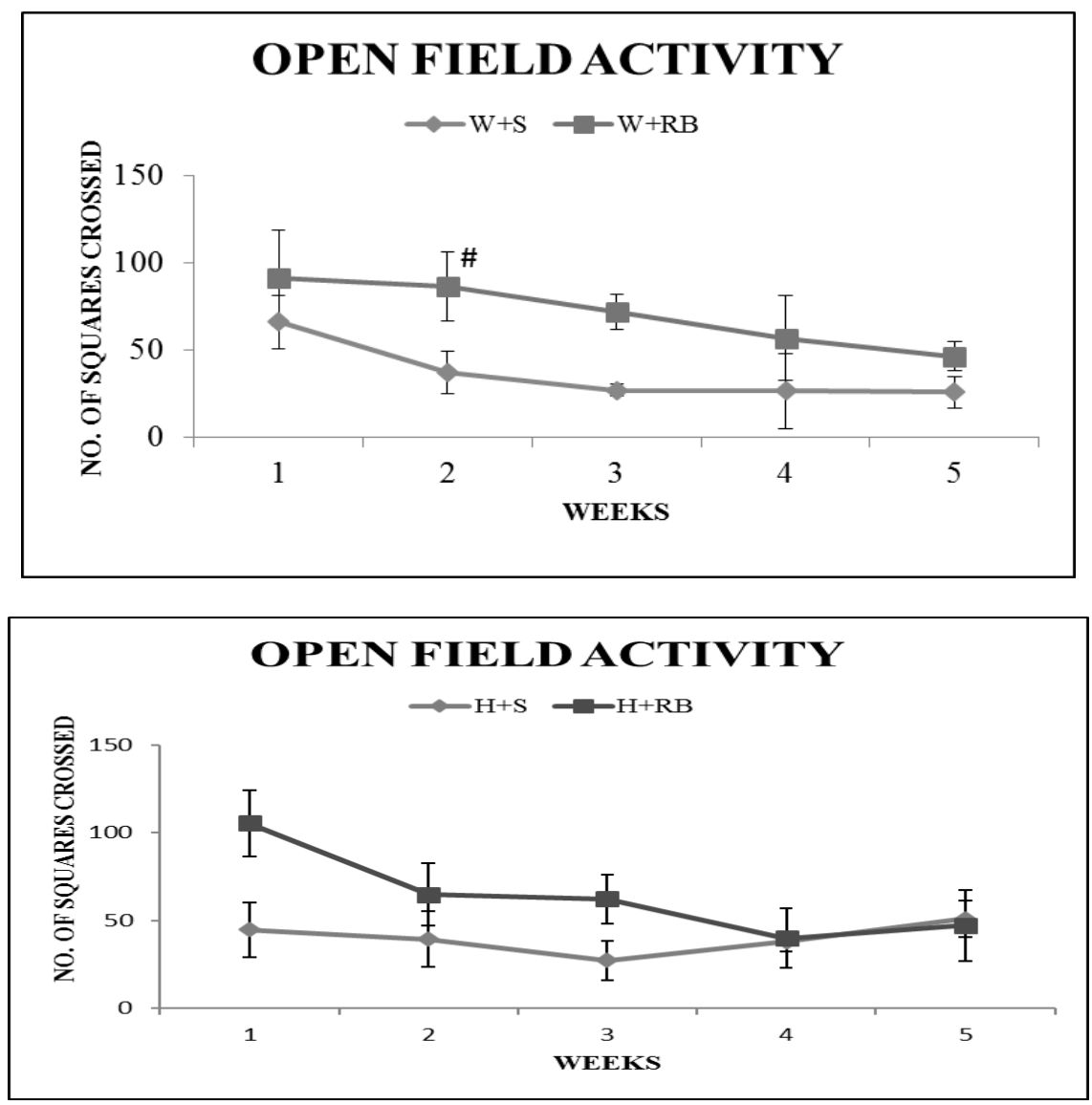

Fig 4.2(a\&b) time course effect of red rice bran oil on haloperidol induced deficits of exploratory activity in an open field

Values are means \pm S.D. $(\mathrm{n}=12)$. Significant differences by Newman-kuels test. ${ }^{*} \mathrm{p}<0.05$ and $* * \mathrm{p}<0.01$ from values of similarly treated animals, $+p<0.05$ and $++p<0.01$ from respective week values of water treated animals and \#p<0.05 and \#\#p<0.01 from respective week values of water+saline and haloperidol+saline treated animals.

Fig $4.3(\mathrm{a} \& \mathrm{~b})$ shows the time course effects of RRBO on haloperidol-induced deficits in Home Cage. Three way ANOVA revealed the significant effects of weeks $(\mathrm{F}=29.309, \mathrm{p}<0.01$, df 8,12), Halo ( $\mathrm{F}=52.884, \mathrm{p}<0.01$, df 8,12), RRBO ( $\mathrm{F}=95.638$, $\mathrm{p}<0.01$, df 8,12 ) and the interaction between weeks*Halo*RRBO $(\mathrm{F}=8.264, \mathrm{p}<0.01$, df 4,12), weeks*halo $(\mathrm{F}=973.227, \mathrm{p}<0.01$, df 4, 12), weeks*RRBO ( $\mathrm{F}=1088.591, \quad \mathrm{p}<0.01$, df 4,12) and Halo*RRBO ( $\mathrm{F}=15162$, $\mathrm{p}<0.01$, df 4,12).

Post hoc analysis by NewmansKeul's test revealed that RRBO alone significantly increased the no. of cages crossed during the $2^{\text {nd }}, 3^{\text {rd }}$ and $4^{\text {th }}$ week of treatment. Treatment of Haloperidol alone significantly decreased the no. of cages crossed in $3^{\text {rd }}$ week but was normalized after that. Whereas the co-administration of Haloperidol with RRBO increased the no. of cages crossed significantly in the $2^{\text {nd }}, 3^{\text {rd }}$ and $4^{\text {th }}$ week of oral administration but decreased drastically during the $5^{\text {th }}$ week of treatment.

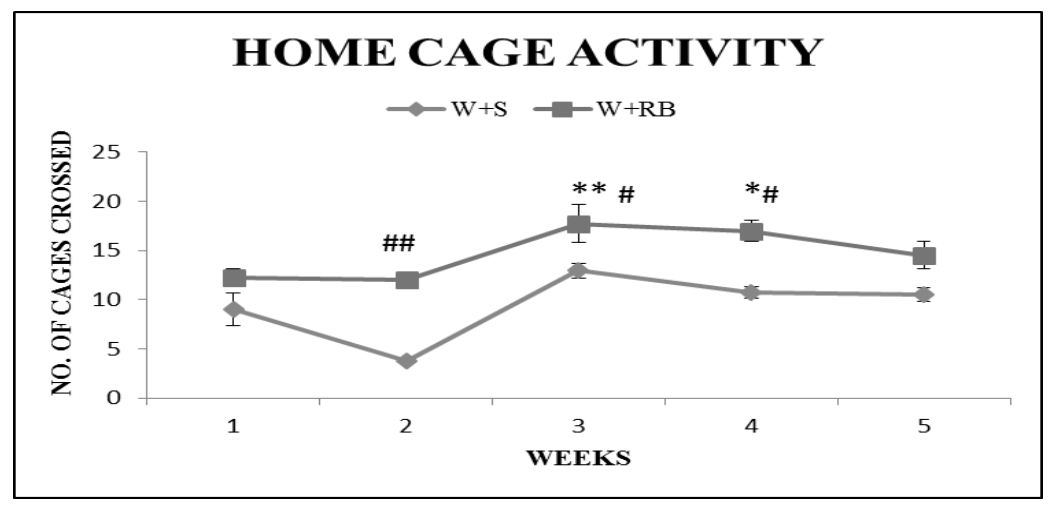




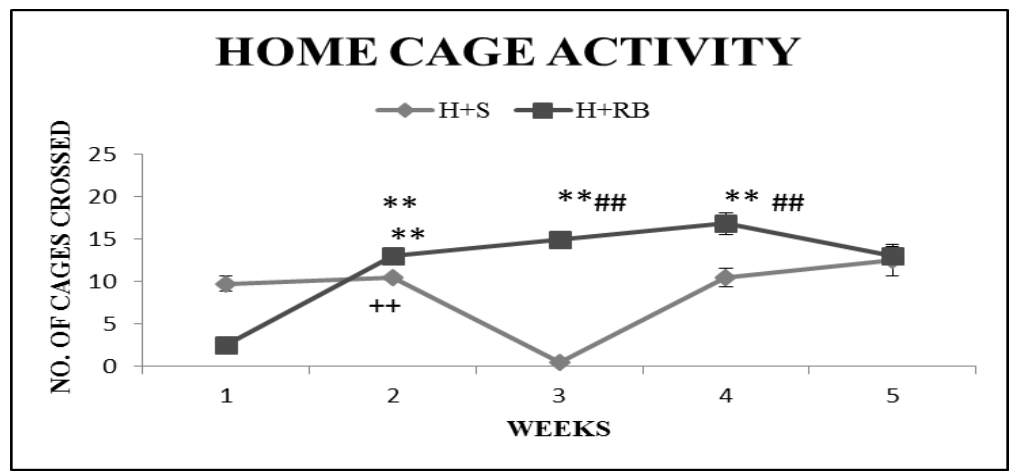

Fig 4. (a\&b) 3 time course effect of red rice bran oil on haloperidol induced Deficits of motor activity in home cage

Values are means \pm S.D. $(n=12)$. Significant differences by Newman-kuels test. $* \mathrm{p}<0.05$ and $* * \mathrm{p}<0.01$ from values of similarly treated animals, $+p<0.05$ and $++p<0.01$ from respective week values of water treated animals and $\# p<0.05$ and \#\#p<0.01 from respective week values of water+saline and haloperidol+saline treated animals.

Fig $4.4(\mathrm{a} \& \mathrm{~b})$ shows the time course effects of RRBO on haloperidol induced deficits in catalepsy. Three way ANOVA showed the significant effects of weeks ( $\mathrm{F}=37.045, \mathrm{p}<0.01,4,12)$, Haloperidol ( $\mathrm{F}=8.825, \mathrm{p}<0.01$, df 8,12), RRBO $(\mathrm{F}=75.596, \mathrm{p}<0.01$, df 8,12), interaction between the weeks*Halo*RRBO ( $\mathrm{F}=60.65$, $\mathrm{p}<0.01$, df 4,12), weeks*Halo ( $\mathrm{F}=38.658$, $\mathrm{p}<0.01$, df 4,12) and weeks*RRBO $(\mathrm{F}=37.203, \mathrm{p}<0.01$, df 4,12). Whereas the interaction between Halo*RRBO ( $\mathrm{F}=1.459$, df 4,12 , NS) was nonsignificant.

RRBO significantly elicited the catalepsy during $1^{\text {st }}, 4^{\text {th }}$ and $5^{\text {th }}$ week of treatment as compared to saline. A gradual tolerance to haloperidol induced catalepsy was observed during 2-5 weeks of drug administration. Co-administration with RRBO significantly decreased the \% catalepsy during 2-5 weeks of oral administration.
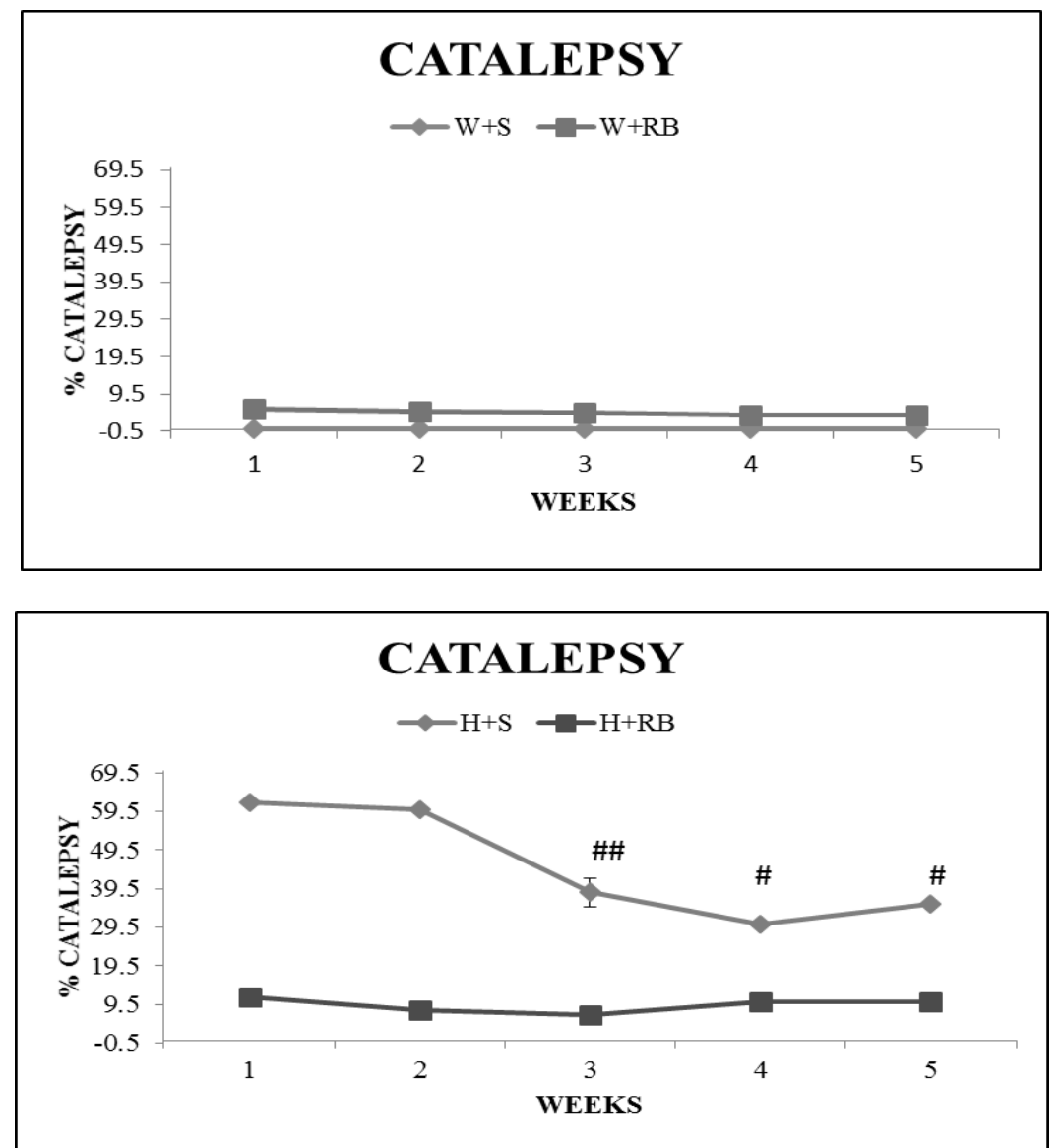

Fig 4.4 $(\mathrm{a} \& \mathrm{~b})$ time course effect of red rice bran oil on haloperidol induced deficits of catalepsy 
Values are means \pm S.D. $(n=12)$. Significant differences by Newman-kuels test. $* p<0.05$ and $* * p<0.01$ from values of similarly treated animals, $+p<0.05$ and $++p<0.01$ from respective week values of water treated animals and $\# p<0.05$ and \#\#p<0.01 from respective week values of water+saline and haloperidol+saline treated animals.

Fig $4.5(\mathrm{a} \& \mathrm{~b})$ shows the time course effects of RRBO on Haloperidol induced deficits in VCMs. Three way ANOVA showed the significant effects of weeks $(\mathrm{F}=150.666, \mathrm{p}<0.01$, df 8,12), Haloperidol ( $\mathrm{F}=178.514, \quad \mathrm{p}<0.01$, df 8,12), RRBO
$(\mathrm{F}=133.907, \mathrm{p}<0.01$, df 8,12), Haloperidol $(\mathrm{F}=133.332, \mathrm{p}<0.01, \mathrm{df}$ $4,12)$, the interaction between weeks*Halo*RRBO $(\mathrm{F}=133.332$, $\mathrm{p}<0.01$, df 4,12), weeks*RRBO ( $\mathrm{F}=155.267$, $\mathrm{p}<0.01$, df 8,12) and Halo*RRBO ( $\mathrm{F}=148.487, \mathrm{p}<0.01$, df 8,12).

Post hoc analysis by NewmansKeul's test depicted that RRBO treated animals did not exhibited any increase in VCMs over control values. Haloperidol administration resulted in significant induction of VCMs in $4^{\text {th }}$ and $5^{\text {th }}$ weeks that increased in a time dependent manner during the subsequent weeks' of drug administration. Animals co-treated with RRBO attenuated Haloperidol induced VCMs in time dependent manner.
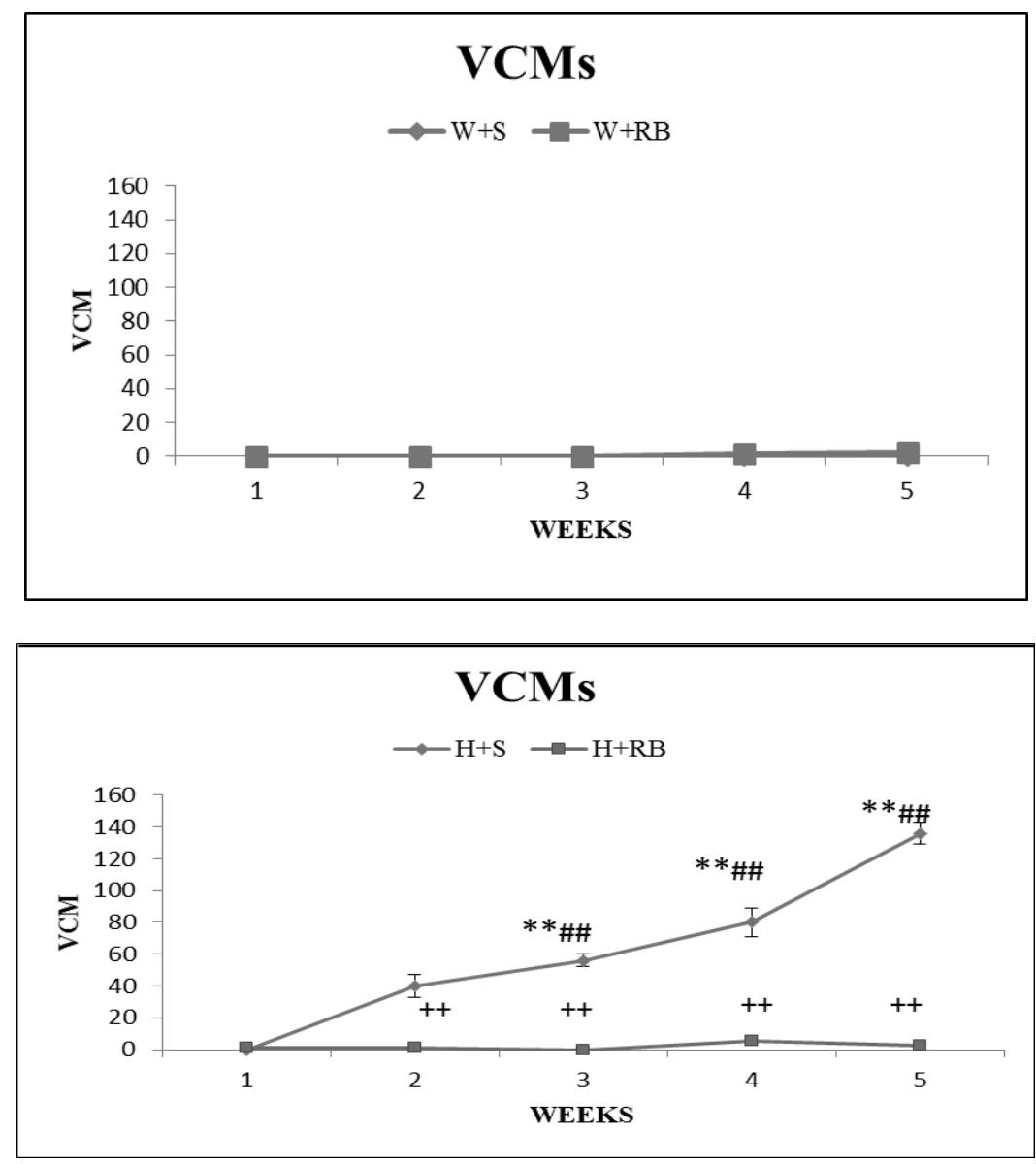

Fig 4.5(a\&b) time course effect of red rice bran oil on haloperidol induced Deficits of vacuous chewing movements

Values are means \pm S.D. $(\mathrm{n}=12)$. Significant differences by Newman-kuels test. ${ }^{*} \mathrm{p}<0.05$ and $* * \mathrm{p}<0.01$ from values of similarly treated animals, $+p<0.05$ and $++p<0.01$ from respective week values of water treated animals and $\# p<0.05$ and \#\#p<0.01 from respective week values of water+saline and haloperidol+saline treated animals.

Fig 4.6(a\&b) showed the time-course effects of RRBO on Haloperidol induced deficits in EPM. Three way ANOVA revealed the significant effects of RRBO ( $F=245.809, \mathrm{p}<0.05$, df 8, 12), significant effects of weeks $(\mathrm{F}=51.156, \mathrm{p}<0.01$, df 4,12$)$,
Haloperidol $(\mathrm{F}=192.835, \mathrm{p}<0.01$, df 8,12), interaction between weeks*halo ( $\mathrm{F}=7.360, \mathrm{p}<0.01$, df 4,12), weeks*RRBO ( $\mathrm{F}=12.966$, $\mathrm{p}<0.01$, df 4,12), weeks*Halo*RRBO ( $\mathrm{F}=39.243$, $\mathrm{p}<0.01$, df 4,12) and Halo*RRBO ( $\mathrm{F}=414.404$, $\mathrm{p}<0.01$, df 4,12 ) were observed.

Post-hoc analysis by NewmansKeul's test revealed that RRBO significantly increased the no of entries in open arm in the $2^{\text {nd }}$ week but was not altered during the $3^{\text {rd }}, 4^{\text {th }}$ and $5^{\text {th }}$ week and Haloperidol alone did not produced any significant effects. The coadministration of haloperidol with RRBO increased the no. of entries during the $3^{\text {rd }}$ and $4^{\text {th }}$ week of treatment significantly. 

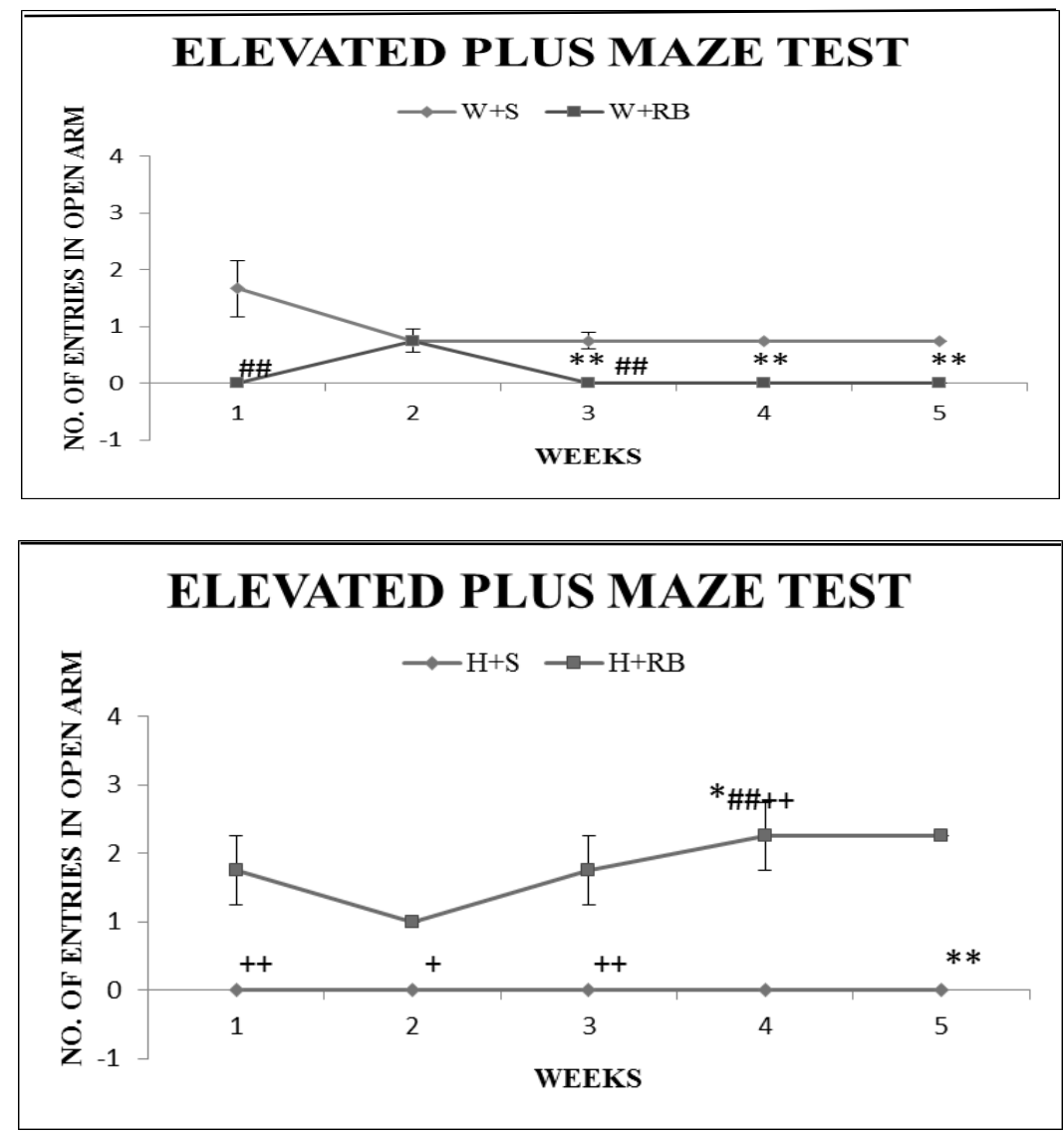

Fig 4.6(a\&b) time course effect of red rice bran oil on haloperidol induced

Anxiety like exploratory behaviour in an elevated plus maze model

Values are means \pm S.D. $(\mathrm{n}=12)$. Significant differences by Newman-kuels test. ${ }^{*} \mathrm{p}<0.05$ and $* * \mathrm{p}<0.01$ from values of similarly treated animals, $+p<0.05$ and $++p<0.01$ from respective week values of water treated animals and $\# p<0.05$ and \#\#p $<0.01$ from respective week values of water+saline and haloperidol+saline treated animals.

Fig $4.7(a \& b)$ shows the time course effects of RRBO on haloperidol induced deficits in EPM. Three way ANOVA revealed the significant effects of weeks $(F=14.525, \mathrm{p}<0.01$, df 4,12) and interaction between weeks*Halo*RRBO $(\mathrm{F}=20.173, \mathrm{p}<0.01$, df $4,12)$ interaction between weeks*Halo $(\mathrm{F}=13.270, \mathrm{p}<0.01$, df 4,12), weeks*RRBO ( $\mathrm{F}=11.387, \mathrm{p}<0.01$, df 4,12) and Halo*RRBO $(\mathrm{F}=26.403$, $\mathrm{p}<0.01$, df 4,12) whereas non-significant effects were observed in Halo ( $\mathrm{F}=0.442$, df 8,12. NS) and RRBO ( $\mathrm{F}=1.933$, df $8,12, \mathrm{NS}$ )

RRBO and Haloperidol alone showed non-significant effects in time spent in open arm throughout the 5 weeks of administration. RRBO co-treated animals exhibited an increase in time spent in open arm as compared to $\mathrm{H}+\mathrm{S}$ treated animals.

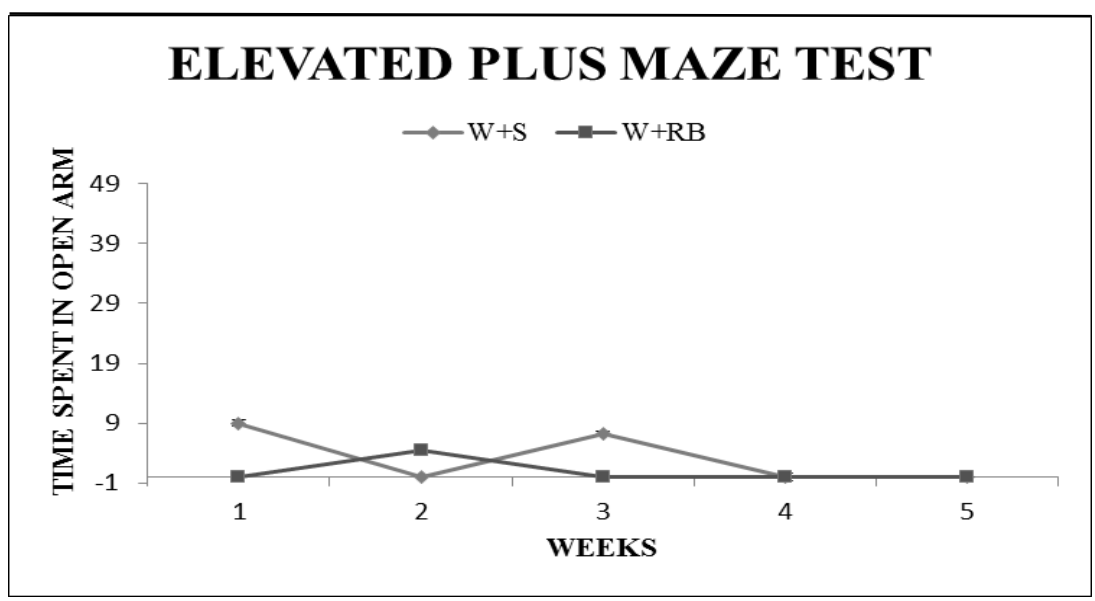




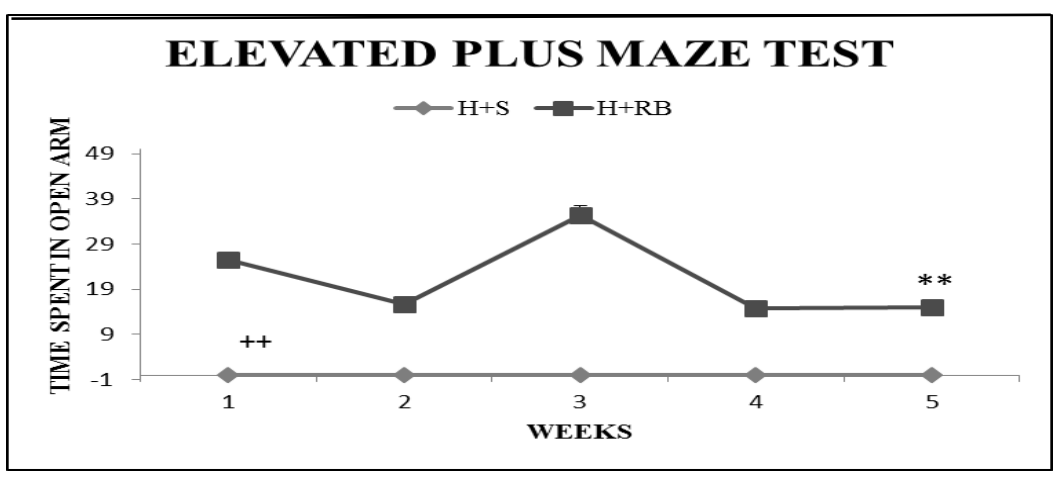

Fig 4.7(a\&b) time course effect of red rice bran oil on haloperidol induced

Anxiety like exploratory behaviour in an elevated plus maze model

Values are means \pm S.D. $(\mathrm{n}=12)$. Significant differences by Newman-kuels test. $* \mathrm{p}<0.05$ and $* * \mathrm{p}<0.01$ from values of similarly treated animals, $+p<0.05$ and $++p<0.01$ from respective week values of water treated animals and $\# \mathrm{p}<0.05$ and \#\#p<0.01 from respective week values of water+saline and haloperidol+saline treated animals.

Fig $4.8(\mathrm{a} \& \mathrm{~b})$ shows the time course effects of RRBO on haloperidol-induced deficits in Light Dark (LD) test. Three way ANOVA showed the significant effects of interaction between weeks*Halo $(\mathrm{F}=6.845, \mathrm{p}<0.01$, df 4,12) and weeks*RRBO $(\mathrm{F}=5.957, \mathrm{p}<0.01$, df 4,12) whereas non-significant effects were observed in weeks' ( $\mathrm{F}=2.036$, df 8,12, NS), Haloperidol ( $\mathrm{F}=1.446$, df 8,12 , NS), RRBO ( $F=0.299$, df 8,12 , NS), interaction between Halo*RRBO ( $\mathrm{F}=0.968$, df 4,12, NS) and weeks*Halo*RRBO $(\mathrm{F}=1.755$, df 4,12 , NS).

Post hoc analysis by NewmansKeul's test showed that Haloperidol alone decreased the no. of entries in light box after the $2^{\text {nd }}$ week but increased significantly in the $5^{\text {th }}$ week of oral administration. RRBO alone altered and increased the no. of entries during the $2^{\text {nd }}$ week but was normalized in $4^{\text {th }}$ week of treatment. When RRBO was co-administered with Haloperidol it showed non-significant effects in the no. of entries in light box.
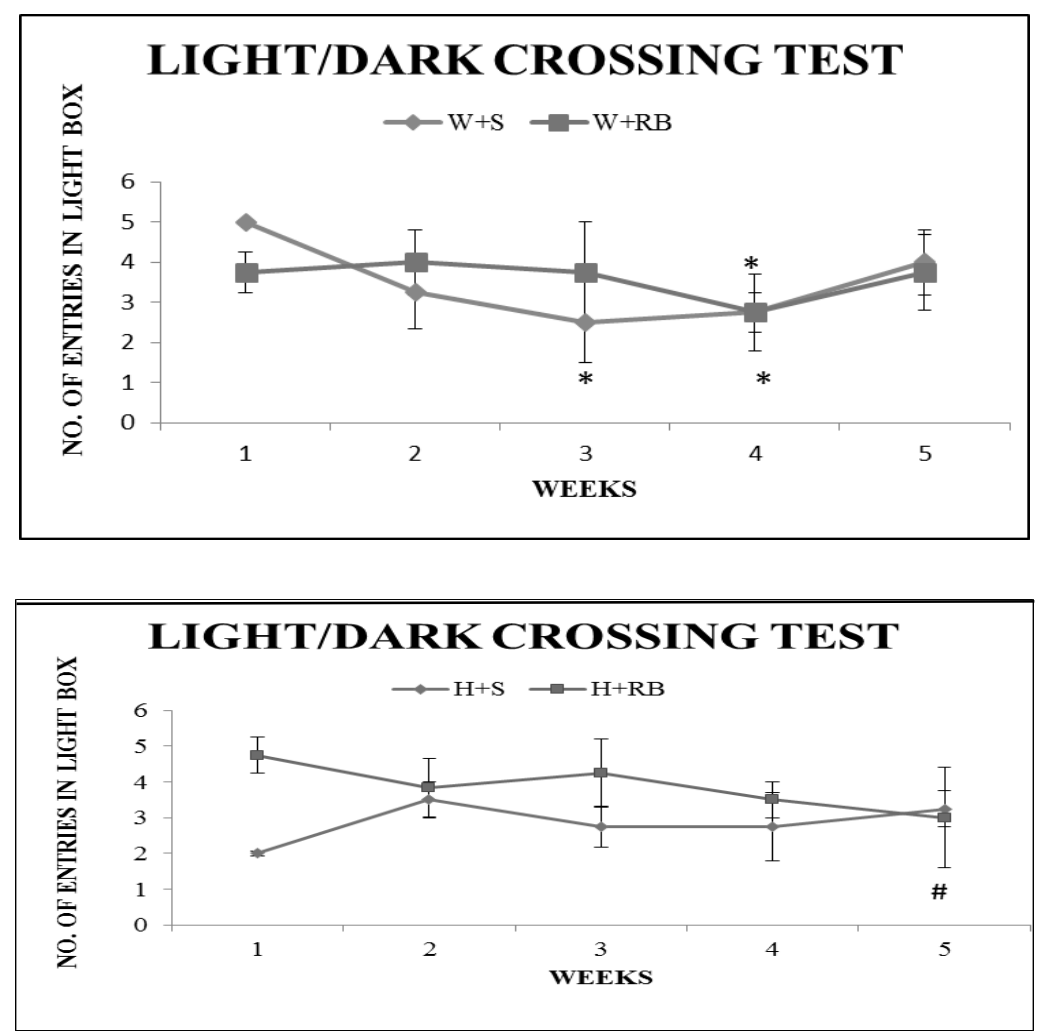

Fig 4.8 $(\mathrm{a} \& \mathrm{~b})$ time course effect of red rice bran oil on haloperidol induced Alteration of light dark compartment activity 
Values are means \pm S.D. $(\mathrm{n}=12)$. Significant differences by Newman-kuels test. $* p<0.05$ and $* * p<0.01$ from values of similarly treated animals, $+p<0.05$ and $++p<0.01$ from respective week values of water treated animals and $\# p<0.05$ and \#\#p<0.01 from respective week values of water+saline and haloperidol+saline treated animals.

Fig $4.9(\mathrm{a} \& \mathrm{~b})$ shows the time course effects of RRBO on haloperidol induced deficits in LD test. Three way ANOVA showed the significant effects of weeks $(\mathrm{F}=6.951, \mathrm{p}<0.01$, df 8,12)

, Haloperidol $(\mathrm{F}=5.753$, $\mathrm{p}<0.01$, df 8,12$)$, RRBO ( $\mathrm{F}=33.505$, $\mathrm{p}<0.01$, df 8,12) and interaction between weeks*Halo $(\mathrm{F}=3.603$, $\mathrm{p}<0.05$, df 4,12). Non significant effects were observed in the interaction between Halo*RRBO ( $\mathrm{F}=0.289$, df 4,12 , NS) and weeks*Halo*RRBO ( $\mathrm{F}=1.703$, df 4, 12, NS).

Post hoc analysis by NewmansKeul's test revealed that Haloperidol alone decreased the time spent in light box and RRBO alone significantly altered the time spent in $3^{\text {rd }}$ and $4^{\text {th }}$ week. Whereas the co-administration with RRBO significantly increased the time spent in LB during $3^{\text {rd }}$ and $4^{\text {th }}$ week of oral administration.
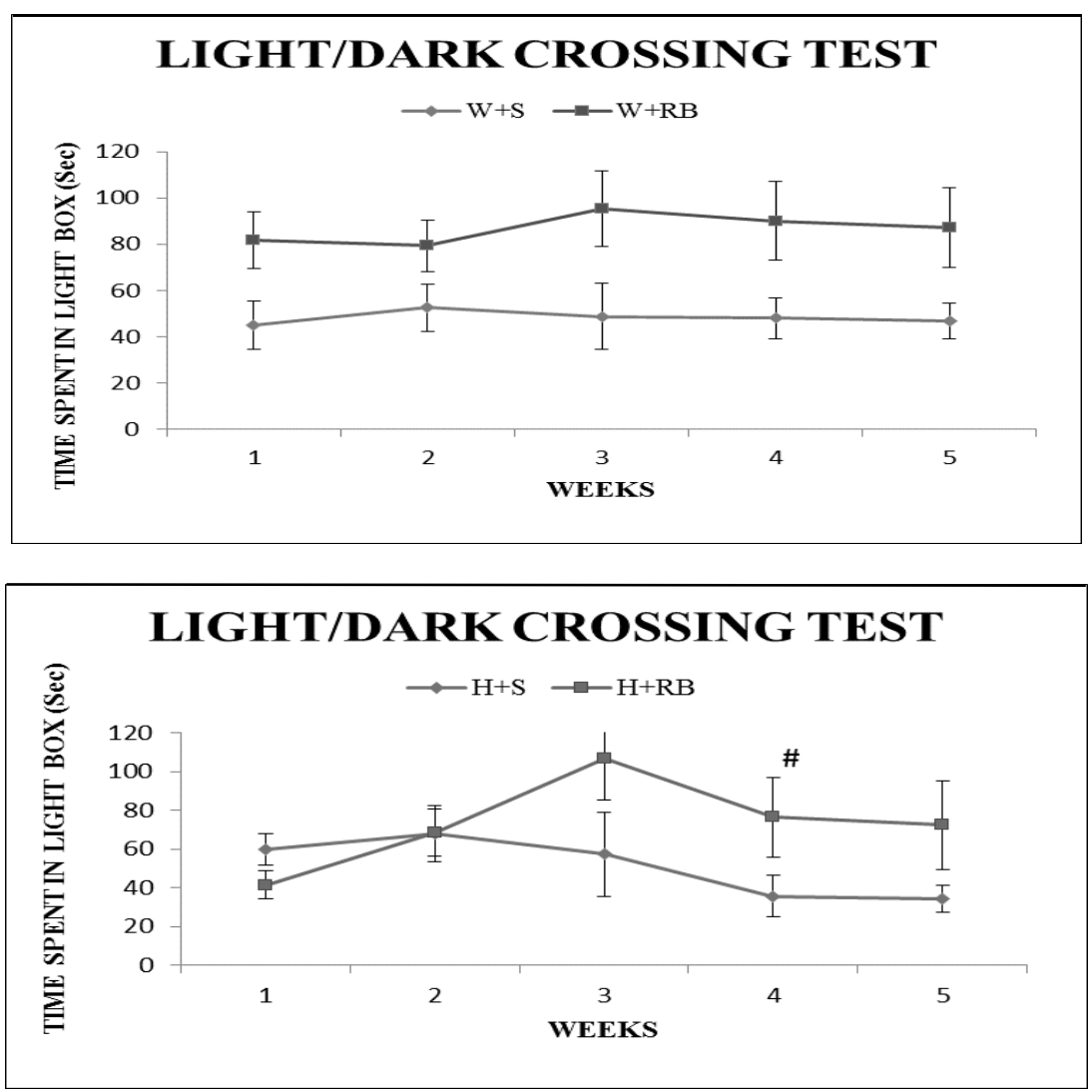

Fig 4.9(a\&b) time course effect of red rice bran oil on haloperidol induced Alteration of light dark compartment activity

Values are means \pm S.D. $(\mathrm{n}=12)$. Significant differences by Newman-kuels test. ${ }^{*} \mathrm{p}<0.05$ and $* * \mathrm{p}<0.01$ from values of similarly treated animals, $+p<0.05$ and $++p<0.01$ from respective week values of water treated animals and $\# \mathrm{p}<0.05$ and $\# \# \mathrm{p}<0.01$ from respective week values of water+saline and haloperidol+saline treated animals.

\section{DISCUSSION}

Our experimental paradigm is principally aimed to study the effects of co-administration of red rice bran oil (RRBO) on the modulation of haloperidol induced anxiety and extra-pyramidal symptoms (EPS). The present study shows that the oral administration of haloperidol at clinically recommended dose produced parkinsonian like symptoms and also VCMs during the five weeks of treatment. An imperative judgment of our current study suggests the reversal of haloperidol induced anxiety and EPS in rats co-treated with RRBO is thought to be associated with free radical scavenging property of oil.

Protective effects of RRBO on haloperidol induced
parkinsonian like effects

The motor activity is controlled and regulated by the dopaminergic system (Petry, 1993; Haleem, 2006; Clausing, 1995) therefore regulatory effects of motor activity are directly related with the DA system. Catalepsy can be described as the inability or difficulty of animal in movement and used as an animal model of parkinsonian like symptoms (Haleem, 2010).

Our results showed that administration of RRBO alone did not elicit catalepsy. On the other hand, coadministration of RRBO 
reversed haloperidol induced cataleptogenic effects. Although, very few data is available regarding the role of RRBO on animal behavior. It has been reported previously the role of stabilized rice bran (SRB) diets on increase in rat exploratory activity in a novel environment in terms of decrease in body weight (Aitken, 2008) and anxiolytic effects of the diet (Jabeen, 2007; Haleem, 1996). Therefore, we can also relate the anxiolytic effect of RRBO with reversal of haloperidol-induced catalepsy.

\section{Protective effects of RRBO on haloperidol induced tardive vems}

Antioxidants neutralize free radicals by donating one of their own electrons, ending the electron-"stealing" reaction. The antioxidant nutrients themselves don't become free radicals by donating an electron because they are stable in either form; they act as scavengers, helping to prevent cell and tissue damage that could lead to cellular damage and disease. These free radicals are widely involved in the production of oxidative stress which eventually produces tardive dyskinesia and VCMs. As the free radicals production and oxidative stress elicited by haloperidol treatment produces TD, therefore it can be conjugated with the previous studies that showed that the haloperidol induced oxidative stress arises from the generation of free radical catecholamine metabolism by monoamine oxidases (MAOs) (Phelan, 2000). Our results showed that rats treated with haloperidol at a clinically recommended dose for 5 weeks displayed VCMs with twitching of facial musculature that increased in a time dependent manner. An important finding of the present study is that the coadministration of RRBO showed a notable reversal of VCMs induced by haloperidol. This finding of current study can be correlated with the anti-oxidative property of RRBO that could prevent the haloperidol induced oxidative stress and results in the engulfment of the free radicals thus treating tardive VCMs.

Thus, we have witnessed an explosion of information on the attenuation and complete reversal of haloperidol induced VCMs by the co-administration of RRBO. It is tempting to relate protective and preventive effects of free radical scavengers RRBO with the reversal of haloperidol induced tardive dyskinesia. It is therefore suggested that incorporation of dietary supplementation like RRBO having antioxidant properties can be useful to develop nutraceuticals for the treatment of schizophrenia.

\section{Protective effects of RRBO on haloperidol induced anxiety}

Anxiety has been described as altered innate aversive behavior as shown in the performance of EPM and LD transitional box activities (see fig. 4.6-4.9) related to diminish phobic condition evident in a variety of rodent studies. Recently, oxidative stress has also been implicated in depression, anxiety disorders and high anxiety levels.

The percentage of time spent in the open arms of the EPM as well as the frequency of entry into the open arms is considered an index of the anxiety levels. In the present study, RRBO alone treated animals did not alter the time spent in the open arm in EPM performance, therefore it is difficult to explain inability of RRBO to change an activity in EPM in terms of its anxiolytic effects. Whereas in LD transitional performance, the treatment of RRBO alone showed anxiolytic parameters suggesting that the long-term dietary $\Omega 3$ PUFA (poly unsaturated fatty acids) supplementation (component of red rice bran oil) positively impacts on anxiety performances in the rats. Therefore, $\Omega 3$ poly unsaturated fatty acids supplementation may be effective in treatment of major depressive disorder (Westbury, 2010).

The results indicated that as the time progressed with haloperidol treatment, a significant decrease was observed in the number of entries and time spent in light box during the $2^{\text {nd }}$ week but increased in the $5^{\text {th }}$ week of haloperidol oral administration which can be correlated with the adaptation to environment. However no significant effects were observed in no. of entries in open arm in an EPM model. The cotreatment with RRBO showed significant anxiolytic properties. Therefore we are the first ones to report the effective role of RRBO on anxiety suggesting its anxiolytic nature when incorporated and administered along with haloperidol.

\section{CONCLUSION}

The present study shows that haloperidol induced parkinsonian like effects following the administration of red rice bran oil were attenuated. This can be explained in terms of positive effects of tocotrienols, tocopherols and omega 3 rich nutrients that are found in red rice bran oil. The present results are consistent with the previous studies which suggest the use of red rice bran oil in nutraceutics as it is thought to be more effective in treating haloperidol induced deficits i.e. parkinsonian like symptoms and tardive dyskinesia. A manifest and remarkable use of red rice bran oil is to treat the haloperidol induced tardive VCMs, thereby attenuating the facial musculature and involuntary movements.

Our present results suggest the reversal of haloperidol induced locomotor deficits and anxiolytic role of red rice bran oil effective in the treatment of TD and PD.

\section{Implications}

The results of our studies suggest the use of neuroleptics adjunctive with those drugs that are rich in sources of omega 3 , as they are helpful in treating the haloperidol induced deficits. For that reason, it can be utilized as normal cooking oil.

However limitations of our studies bound us to narrate any of the changes occurring at the neurochemical level as we only dealt with behavioral aspects and its effects after the administration of RRBO on locomotion and anxiety. Further studies can be carried out in this regard suggesting the effects of RRBO in brain dopamine and serotonin levels. Furthermore, clinical trials can be done to confirm the hypothesis of its usage for human consumption.

\section{REFERENCES}

- Alonso, J., \&Lépine, J. P. (2007). Study of the Epidemiology of Mental Disorders/Mental Health Disability: A European Assessment in the Year 2000 Scientific Committee. Journal of Clinical Psychiatry, 68(2), 3-9.

- Addington, J., Cadenhead, K. S., Cannon, T. D., Cornblatt, B., McGlashan, T. H., Perkins, D. O., \& Heinssen, R. (2007). North American Prodrome Longitudinal Study: a collaborative multisite approach to prodromal schizophrenia research. Schizophrenia Bulletin, 33(3), 665-672. 
- $\quad$ Aitken, R. J., \& Roman, S. D. (2008). Antioxidant systems and oxidative stress in the testes. Oxidative medicine and cellular longevity, 1(1), 15-24.

- Jabeen, B., Badaruddin, M., Ali, R., \& Haleem, D. J. (2007). Attenuation of restraint-induced behavioral deficits and serotonergic responses by stabilized rice bran in rats. Nutritional neuroscience, 10(1-2), 11-16.

- Clausing, P., Gough, B., Holson, R. R., Slikker, W., \& Bowyer, J. F. (1995). Amphetamine levels in brain microdialysate, caudate/putamen, substantia nigra and plasma after dosage that produces either behavioral or neurotoxic effects. Journal of Pharmacology and Experimental Therapeutics, 274(2), 614-621.

- Dilsaver, S. C., \& Alessi, N. E. (1988). Antipsychotic withdrawal symptoms: phenomenology and pathophysiology. Acta Psychiatrica Scandinavica, 77(3), 241246.

- Halliwell, B. (2006). Oxidative stress and neurodegeneration: where are we now?. Journal of neurochemistry, 97(6), 16341658.

- Haleem, D. J., \& Batool, F. (1996). Regionally specific effects of diazepam on brain serotonin metabolism in rats: sustained effects following repeated administration. Life sciences, 59(15), 239-246.

- Haleem, D. J., Batool, F., Khan, N. H., Kamil, N., Ali, O., Saify, Z. S., \& Haleem, M. A. (2002). Differences in the effects of haloperidol and clozapine on brain serotonin and dopamine metabolism and on tests related to extrapyramidal functions in rats. Medical Science Monitor, 8(9), 354-361.

- Haleem, D. J., Samad, N., \& Haleem, M. A. (2007). Reversal of haloperidol-induced extrapyramidal symptoms by buspirone: a time-related study. Behavioural pharmacology, 18(2), 147-153.
- Haleem, D. J., Samad, N., \& Haleem, M. A. (2007). Reversal of haloperidol-induced tardive vacuous chewing movements and supersensitive somatodendritic serotonergic response by buspirone in rats. Pharmacology Biochemistry and Behavior, 87(1), 115-121.

- Haleem, D. J. (2006). Serotonergic modulation of dopamine neurotransmission: a mechanism for enhancing therapeutics in schizophrenia. Journal of the College of Physicians and Surgeons--Pakistan, 16(8), 556-562.

- Marchese, G., Casu, M. A., Bartholini, F., Ruiu, S., Saba, P., Gessa, G. L., \& Pani, L. (2002). Sub-chronic treatment with classical but not atypical antipsychotics produces morphological changes in rat nigro-striatal dopaminergic neurons directly related to 'early onset' vacuous chewing. European Journal of Neuroscience, 15(7), 1187-1196.

- Phelan, J. C., Link, B. G., Stueve, A., \& Pescosolido, B. A. (2000). Public conceptions of mental illness in 1950 and 1996: What is mental illness and is it to be feared?. Journal of Health and Social Behavior, 41(2), 188-207.

- $\quad$ Petry, N., Furmidge, L., Tong, Z. Y., Martin, C., \& Clark, D. (1993). Time sampling observation procedure for studying interaction between d-amphetamine and selective DA receptor in the rats. Pharmacology Biochemistry and Behavior, 44(1), 167-180.

- Westbury, J., Jackson, S., Gee, P., \& Peterson, G. (2010). An effective approach to decrease antipsychotic and benzodiazepine use in nursing homes: the RedUSe project. International Psychogeriatrics, 22(01), 26-36. 